Meta

Journal des tradlucteurs

Translators' Journal

\title{
Traduction médicale
}

\section{Jacques Boulay}

Volume 13, numéro 3, septembre 1968

URI : https://id.erudit.org/iderudit/002122ar

DOI : https://doi.org/10.7202/002122ar

Aller au sommaire du numéro

Éditeur(s)

Les Presses de l'Université de Montréal

ISSN

0026-0452 (imprimé)

1492-1421 (numérique)

Découvrir la revue

Citer cet article

Boulay, J. (1968). Traduction médicale. Meta, 13(3), 127-132.

https://doi.org/10.7202/002122ar

Ce document est protégé par la loi sur le droit d'auteur. L'utilisation des services d'Érudit (y compris la reproduction) est assujettie à sa politique d'utilisation que vous pouvez consulter en ligne.

https://apropos.erudit.org/fr/usagers/politique-dutilisation/
Cet article est diffusé et préservé par Érudit.

Érudit est un consortium interuniversitaire sans but lucratif composé de l’Université de Montréal, l'Université Laval et l'Université du Québec à Montréal. Il a pour mission la promotion et la valorisation de la recherche. https://www.erudit.org/fr/ 


\section{PROBLÈMES ET SOLUTIONS}

\section{TRADUCTION MÉDICALE}

J'ai eu récemment l'occasion d'étudier les textes français et anglais d'un emballage de la maison Becton, Dickinson \& Co. et j'ai pensé que les lecteurs de la revue Meta pourraient être intéressés à connaître les problèmes de langue que peut soulever ce genre de texte.

Disons tout de suite que l'emballage en question est une boîte de carton et qu'il contient des aiguilles qui peuvent être jetées après usage. Les figures 1 et 2 sont la reproduction de deux côtés symétriques de la boîte et c'est par eux que nous commencerons notre étude.

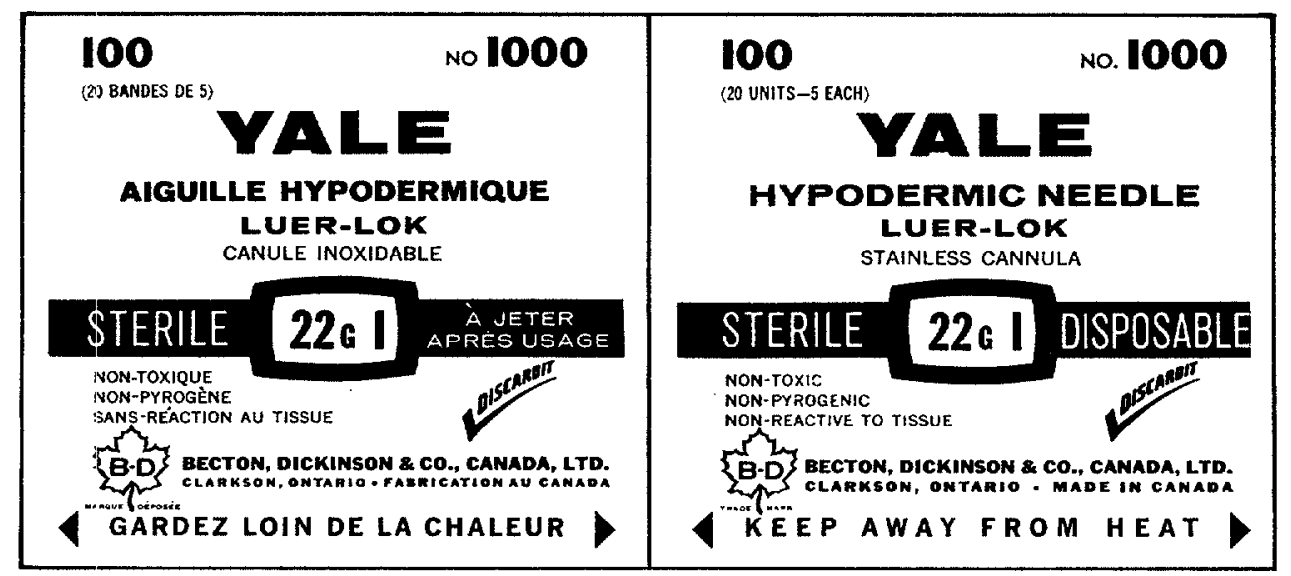

Fig. 1

Fig. 2

$1^{\circ}$ Il y a 100 aiguilles par boîte. Chaque aiguille est placée dans une petite enveloppe de plastique assez rigide qui épouse quelque peu sa forme, et cinq de ces petites enveloppes sont accolées les unes aux autres. L'ensemble est désigné, 
dans le texte anglais, du nom passe-partout de unit. Le traducteur a proposé le mot «bande», qui ne convient certainement pas, puisqu'il n'évoque qu'une caractéristique secondaire de l'objet, c'est-à-dire sa forme rectangulaire allongée. Le caractère le plus important de cet objet, c'est d'être une enveloppe, et une enveloppe spéciale qui isole les aiguilles les unes des autres en s'adaptant plus ou moins à leur forme. Le mot français qui, me semble-t-il, convient le mieux est étui. Robert définit le mot étui de la manière suivante: enveloppe le plus souvent rigide, dont la forme, la disposition est adaptée à l'objet, aux objets qu'elle est destinée à contenir.

$2^{\circ} \mathrm{N}^{\circ} 1000$ devrait s'écrire, en bonne typographie, Numéro 1000. En effet, en dehors de son emploi comme subdivision d'ouvrage, le mot numéro, suivi d'un nombre, ne s'abrège que s'il est immédiatement précédé du mot auquel il se rapporte (Code typographique, p. 13).

$3^{\circ}$ Yale est une marque de commerce. Le mot doit donc être identique en français et en anglais.

$4^{\circ}$ L'expression aiguille hypodermique, bien qu'elle se superpose exactement à l'expression anglaise hypodermic needle, est tout à fait correcte.

$5^{\circ}$ Quant à Luer-Lok, c'est un terme qui désigne le procédé par lequel les aiguilles se fixent à la seringue. Je dois à l'obligeance du représentant à Québec de la firme Becton, Dickinson \& Co. les renseignements suivants sur l'origine de ce terme. Chose curieuse, il vient du nom d'une société parisienne, la société H. Wolfing Luer, dont un employé, Carl Schneider, inventa en 1896 la première seringue entièrement en verre. Quelque temps plus tard, la société Luer obtenait un brevet américain pour cette seringue et, en 1898, elle vendait ses droits à Becton-Dickinson. À cette époque, il existait plusieurs sortes d'embouts de seringue, qui différaient les uns des autres par la forme et les dimensions. Le mot Luer en arriva rapidement à désigner un embout bien particulier et, durant le premier quart du $\mathrm{xx}^{\mathrm{e}}$ siècle, cet embout devint le plus communément utilisé aux États-Unis. En 1925, Becton-Dickinson inventait un procédé nouveau de fixation de l'aiguille à la seringue grâce à un épaulement qui termine l'embout de l'aiguille et qui permet de visser celle-ci à l'intérieur d'un embout de seringue fileté. Ce dispositif fut dénommé Luer-Lok (Lok étant une simplification publicitaire de Lock).

$6^{\circ}$ Stainless cannula a été traduit en français par «canule inoxidable». Notons d'abord qu'il faut écrire inoxydable et non «inoxidable». Mais il ne s'agit là que d'une faute d'orthographe. Par contre, il est plus grave de parler de la «canule» d'une aiguille au lieu de la tige d'une aiguille. Les aiguilles hypodermiques comprennent une tige tubulée et un embout. Le mot «canule», en français, désigne un petit tuyau que l'on adapte à l'extrémité d'une seringue, d'un tube à injection, d'une poire à lavement.

$7^{\circ} \dot{A}$ jeter après usage est une traduction fort satisfaisante de disposable. Mais l'expression est un peu longue et le Comité d'étude des termes médicaux français Clair-Dire, ainsi que le Comité d'étude des termes de médecine du Québec recommandent le terme jetable, qui a l'avantage de pouvoir s'employer comme adjectif et comme substantif. On peut se servir aussi de l'expression non récupérable pour traduire disposable. Certains préconisent également uni-service, surtout pour des raisons de psychologie publicitaire, jetable pouvant avoir une nuance 
péjorative. À remarquer que disposer veut dire posséder: si je dispose d'une voiture, c'est que $\mathrm{j}$ 'ai une voiture. Tandis que to dispose veut dire ne plus posséder, se débarrasser.

$8^{\circ}$ Non-toxic et non-pyrogenic peuvent se traduire par atoxique et apyrogène. On aurait pu aussi combiner les deux idées de la façon suivante: sans action toxique ou pyrogène. Quant à non-reactive to tissue, il est difficile de savoir si cela veut dire que l'aiguille n'agit pas sur les tissus ou que les tissus n'agissent pas sur l'aiguille. C'est probablement la première idée qui est la bonne, puisqu'on ne s'imagine pas facilement pourquoi les utilisateurs pourraient être préoccupés de l'action des tissus sur l'aiguille, celle-ci devant être jetée après usage. La traduction française "sans réaction au tissue » est probablement un contre-sens et il aurait fallu dire sans action tissulaire. À noter que tissu ne s'écrit pas «tissue » en français.

Keep away from heat a été traduit littéralement par "gardez loin de la chaleur». Même si, grammaticalement et lexicalement, cette phrase n'est pas fautive, ce n'est pas celle que l'usage français prescrit d'employer. En français international, on rend cette idée par l'expression craint la chaleur. Comme il arrive souvent, le français, à cause de sa préférence pour le plan de l'abstrait, réussit à rendre une idée en moins de mots ou de syllabes que l'anglais (quatre syllabes en français pour cinq en anglais ).

Le texte de la figure 2 pourrait donc se rendre de la façon suivante:

20 étuis de 5 aiguilles

$$
\text { Y A L E }
$$

Numéro 1000

$$
\begin{gathered}
\text { Aiguilles hypodermiques } \\
\text { LUER-LOK } \\
\text { à tige inoxydable }
\end{gathered}
$$

\section{STÉRILE}

$$
22 \mathrm{G} 1
$$

JETABLE

Sans action toxique ou pyrogène Sans action tissulaire

$$
\begin{gathered}
\text { C }^{\text {ie }} \text { Becton \& Dickinson (Canada) Limitée } \\
\text { Clarkson, Ontario } \\
\text { Fabriquées au Canada } \\
\text { CRAINT LA CHALEUR }
\end{gathered}
$$

Les fígures 3 et 4 représentent les deux autres côtés symétriques de la boîte.

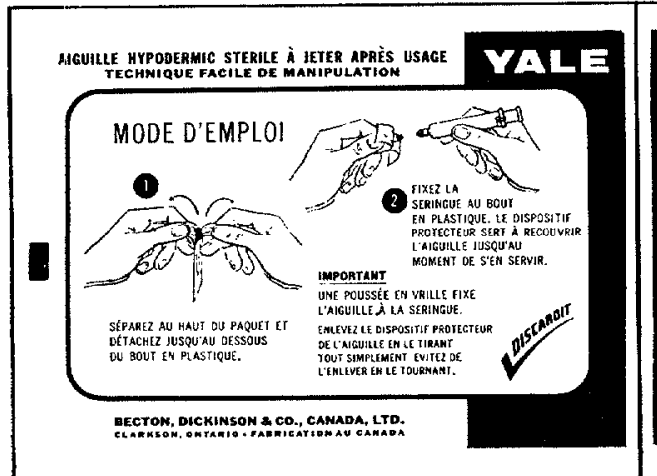

Fig. 3

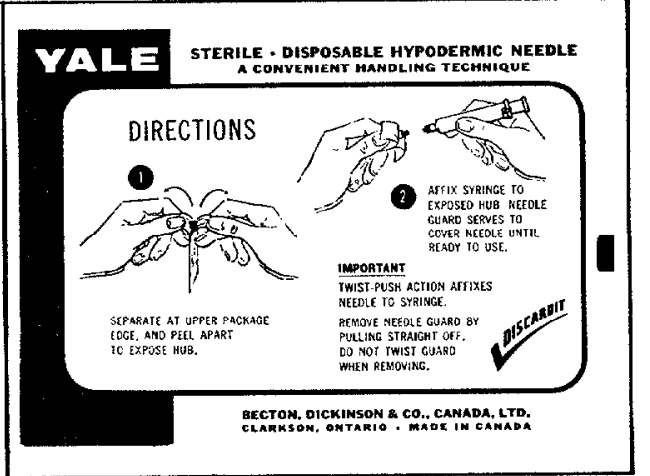

Fig. 4 
$1^{\circ}$ Sterile. Disposable hypodermic needle pourrait se traduire par aiguilles stériles jetables. En effet, il n'est pas nécessaire de dire ici qu'il s'agit d'aiguilles hypodermiques, puisque la chose a été mentionnée ailleurs sur l'emballage et que toutes les aiguilles à injection sont en fait des aiguilles hypodermiques. À noter qu'hypodermic se rend en français par hypodermique et non par «hypodermic», et que stérile prend un accent aigu dans notre langue.

$2^{\circ}$ 《Technique facile de manipulation» ne veut strictement rien dire. Comment une technique pourrait-elle être facile de manipulation? Une technique peut être facile ou difficile à mettre en œuvre, à mettre au point, à réaliser, à exécuter; elle peut comporter des manipulations difficiles, mais elle ne peut pas être elle-même de manipulation difficile. Le texte anglais est lui-même fort imprécis: dans a convenient handling technique, on ne veut certainement pas dire que l'aiguille est une technique, mais que la manipulation de cette aiguille est facile et que son emploi rend aisée la technique des injections hypodermiques. À mon avis, il faudrait combiner les deux phrases et ajouter simplement à aiguilles stériles jetables les qualificatifs commode et de maniement facile.

$3^{\circ} \mathrm{La}$ première partie du mode d'emploi appelle les remarques suivantes. Le texte anglais dit: Separate at upper package edge, and peel apart to expose hub, ce qu'on a traduit en français par « Séparez au haut du paquet et détachez jusqu'au dessous du bout en plastique ». C'est gauche et pénible. Je suggérerais, pour ma part: Dégagez l'embout de l'aiguille en ouvrant le haut de l'enveloppe qui la recouvre. L'illustration est suffisamment claire pour qu'on n'ait pas à expliquer comment on doit ouvrir l'enveloppe (to peel apart).

$4^{\circ} \mathrm{Au}$ numéro 2 , le texte anglais est lui-même déficient. Si l'on inclut ce qu'il y a sous la rubrique Important, ce texte comprend quatre phrases: la première et la troisième portent sur la technique de fixation de l'aiguille à la seringue, la deuxième et la quatrième parlent de la gaine qui recouvre l'aiguille. Il serait beaucoup plus logique de grouper ensemble la première et la troisième phrase, puis ensuite la deuxième et la quatrième. Affix syringe to exposed hub. Twist-push action affixes needle to syringe pourrait se traduire alors de la manière suivante: Insérez la seringue dans l'embout de l'aiguille et serrez à bloc en vissant, plutôt que par «Fixez la seringue au bout de plastique. Une poussée en vrille fixe l'aiguille à la seringue ». Le regroupement des deuxième et quatrième phrases pourrait de son côté se traduire par: Une gaine en plastique recouvre l'aiguille et la protège jusqu'au moment de l'injection. Pour l'enlever, il suffit de tirer sur elle dans l'axe de l'aiguille, sans tourner. Cela remplacerait assez avantageusement les deux phrases françaises proposées par le traducteur, qui sont longues et qui contiennent quelques incorrections. Par exemple, needle guard se rend en français par gaine et non par «dispositif protecteur»: il s'agit en effet d'une gaine en plastique qui recouvre entièrement l'aiguille pour éviter qu'elle ne soit contaminée avant le moment de l'injection; cette gaine adhère - " entre à serre », pour employer un canadianisme de bon aloi - à la portion inférieure de l'embout et il faut exercer une certaine traction pour l'en détacher. Par ailleurs, la gaine ne sert pas à recouvrir l'aiguille, elle la recouvre tout simplement. On pourrait dire qu'elle sert à recouvrir l'aiguille, si elle était séparée de celle-ci, mais elle ne l'est pas. 
Il n'est pas surprenant que le langage des professionnels de la santé soit mauvais, quand on songe qu'ils ont sous les yeux à longueur de journée des textes comme celui-ci, qui sont traduits de l'anglais par des traducteurs incompétents, qui ignorent à peu près tout de la langue française. Il devrait d'ailleurs y avoir une loi pour obliger tout traducteur d'un texte anglais à signer son travail. Le cas échéant, on saurait donc qui stigmatiser.

Pour mieux conduire cette étude, j'ai dépouillé quelques ouvrages français dans le but d'accumuler autant de documentation que possible sur les injections et le matériel qu'on emploie pour les faire. Voici les termes que j'ai notés et qui pourraient constituer un de ces jours l'embryon d'un véritable vocabulaire.

Injections hypodermiques - Elles comprennent les injections sous-cutanées, les injections intraveineuses, les injections intradermiques, les injections intramusculaires.

Seringue - Seringue en verre; seringue en plastique, seringue du modèle de Luer; contenance d'une seringue; seringue genre Barthélemy (avec graduation précise au $1 / 10 \mathrm{de}^{\mathrm{cm}}{ }^{3}$ ); remplissage d'une seringue, éliminer les bulles d'air d'une seringue, seringue remplie; seringue munie de son aiguille; la seringue est formée de deux pièces (un corps de pompe et un piston); monter la seringue et l'aiguille; démonter la seringue.

Matériel - Matériel pour injection hypodermique; plateau (où l'on a déposé le matériel); matériel qui n'est utilisable qu'une fois.

Corps de pompe - Corps de pompe gradué; graduation du corps de pompe; corps de pompe armé à ses deux extrémités d'une garniture métallique soudée.

Piston - Piston creux; piston rodé; piston qui glisse à frottement doux dans le corps de la pompe; piston métallique; bouton du piston; pousser le piston.

Embout - Embout de seringue; embout de Luer; verrou américain (Luer-Lok); embout rodé; embout-raccord; embout-raccord métallique; embout-raccord en matière plastique.

Aiguilles - Aiguilles (en acier, en nickel, en platine irridié); longueur d'une aiguille; calibre d'une aiguille; aiguille fine; grosse aiguille; pavillon d'une aiguille; tige tubulée d'une aiguille; nettoyage des aiguilles; stérilisation des aiguilles; conservation des aiguilles; affûtage des aiguilles; le liquide doit affleurer au haut de l'aiguille; rupture d'une aiguille; embout d'une aiguille; embout d'une aiguille terminé par un épaulement; aiguille jetable.

Ailettes - Ailettes qui servent de point d'appui aux doigts pour pousser aisément le piston au moment de l'injection; ailettes latérales d'une aiguille.

Mandrin - Fil métallique introduit par le pavillon jusqu'à la pointe. Mandrin qui assure l'intégrité de la lumière; seringue débarrassée de son fil-mandrin; mandrin piquant.

Trocart - Trocart métallique; trocart mousse.

Garrot - Pince pour fixer le garrot; application du garrot.

Pince - Pince presse-tube; pince de Mohr.

Biseau - Biseau court, moyen ou long.

Ampoules — Scieuse destinée à ouvrir les ampoules. 
Pavillon - Pavillon s'adaptant à l'embout de la seringue; pavillon s'adaptant au raccord; partie externe du pavillon de l'aiguille.

Boîte - Boîte d'urgence; boîte métallique garnie d'un coussin de gaze ou de coton cardé.

$D^{\mathrm{r}}$ JaCQues BoulaY 